\title{
In Situ Silver Decoration on Graphene Oxide-Treated Thin-Film Composite Forward Osmosis Membranes: Biocidal Properties and Regeneration Potential
}

\author{
Environmental Science and Technology Letters
}

Revised: December 04, 2015

Adel Soroush ${ }^{1}$, Wen $\mathrm{Ma}^{1}$, Myriam Cyr ${ }^{1}$, Md. Saifur Rahaman ${ }^{1 *}$, Bahareh Asadishad ${ }^{2}$, Nathalie Tufenkji ${ }^{2}$

${ }^{I}$ Department of Building, Civil and Environmental Engineering, Concordia University

Montreal, Quebec, Canada, H3G 1 M8

${ }^{2}$ Department of Chemical Engineering, McGill University,

Montreal, QC, Canada H3A OC5

${ }^{*}$ Corresponding author. Tel.: +15148482424, Ext. 5058

E-mail address: saifur.rahaman@concordia.ca (M.S. Rahaman) 


\begin{abstract}
The surfaces of thin film composite (TFC) forward osmosis (FO) membranes were modified by in situ formation of silver nanoparticles (AgNPs) in the presence and absence of graphene oxide (GO) nanosheets to impart biocidal properties to the membranes. The abundance of oxygen-containing functional groups in GO makes it suitable for anchoring $\mathrm{Ag}^{+}$ions and governing the size, shape, and distribution of AgNPs. The presence of GO resulted in the formation of smaller and uniformly distributed AgNPs as well as increased silver loading, higher stability, and enhanced ion-release control. Membranes modified by both GO and Ag exhibited improved (98\%) bacterial inactivation when compared to only Ag-modified (80\%) or GO-modified membranes (50\%). After seven days of Ag ion release from GO-Ag-modified membranes, AgNP regeneration was conducted identically to the in situ Ag formation procedure. After regeneration, the membrane regained nearly all of its antibacterial properties and $75 \%$ of its initial silver loading.
\end{abstract}

\title{
1. INTRODUCTION
}

With increasing application of membrane-based water treatment and desalination processes, ${ }^{1-}$ ${ }^{3}$ attention to fouling and biofouling as major limitations of this technology is increasing accordingly. Amongst the different methods of fouling and biofouling mitigation, membrane surface modification using different modifiers and procedures is receiving considerable recognition as an effective and flexible approach. ${ }^{4-6}$

Silver nanoparticles (AgNPs) are very effective biocidal NPs and have been investigated regarding their formation mechanisms, ${ }^{7-10}$ biocidal properties, ${ }^{11-13}$ and their applicability in either membrane surface modification ${ }^{14-19}$ or membrane bulk incorporation. ${ }^{20-22}$ Although AgNPs provide significant and effective biocidal properties, their intrinsic tendency to aggregate, and their fast dissolution or release in aqueous media have raised concerns as to their long-term efficacy and the possibility of regeneration. ${ }^{23}$ The combination of AgNPs and carbon-based nanomaterials introduces a new class of emerging nanomaterials which offer physical durability and more effective biocidal properties. ${ }^{24,25}$

Among a variety of carbon-based materials, graphene oxide (GO) nanosheets have attracted significant attention because of their unique physical and chemical properties. ${ }^{26}$ Oxygen- 
containing functional groups of $\mathrm{GO}^{27}$ can serve as anchors for the AgNP nuclei and govern their shape, size, and distribution. GO nanosheets also show extensive biocidal properties and toxicity towards microorganisms ${ }^{28-30}$, thus increasing the efficacy of the biocidal properties of GO-Ag nanohybrids. ${ }^{31}$ GO-Ag nanocomposites, among other types of nanohybrids, have attracted attention for antimicrobial applications, ${ }^{32-34}$ and more recently, for the functionalization of nanofiber mats ${ }^{35}$ and thin film composite (TFC) forward osmosis (FO) membranes. ${ }^{36}$

To the best of our knowledge, our recent publication reports for the first time, surface modification of $\mathrm{FO}$ membranes by $\mathrm{GO} / \mathrm{Ag}$ nanocomposites. ${ }^{36}$ Although GO-Ag nanocomposites present strong biocidal properties, their application still suffers from drawbacks such as the difficulty of synthesis, limited Ag loading, and lack of regeneration potential. Therefore, this current study focuses on using GO nanosheets as a sublayer modifier for in situ AgNP formation on TFC FO membranes to overcome the aforementioned limitations. The new modification procedure provides a covalent bond between GO and membrane surface which is permanent in comparison to the covalent bond between silver and the membrane surface in our pervious study. The effects of the presence of GO on the loading and release of silver, membrane surface hydrophilicity, biocidal properties, and membrane performance were investigated. Finally, the potential of AgNP regeneration after depletion in water for seven days, for the first time, was investigated.

\section{MATERIALS AND METHODS}

\subsection{Materials}

The following chemicals were used as received from Sigma-Aldrich: Silver nitrate (99.9999\% trace metal basis), N-(3-Dimethylaminopropyl)-N'-ethylcarbodiimide hydrochloride (EDC), N-Hydroxysuccinimide (NHS), Ethylenediamine (ED), MES and HEPES buffer, and sodium borohydride (99.99\%). The sample of graphene oxide was purchased from Cheap Tubes Company (Brattleboro, USA). Deionized (DI) water was prepared in a Millipore Milli-Q purification system. The polyamide (PA) TFC FO membranes were obtained from HTI and were soaked in DI water for 24 hrs before modification. 


\subsection{In situ AgNP formation on GO modified membranes}

Graphene oxide nanosheets were covalently bonded to the surface of membranes through the formation of amine reactive esters. AgNPs were then formed in situ on the surface of GOmodified membranes through the wet chemical reduction of $\mathrm{AgNO}_{3}$ by $\mathrm{NaBH}_{4}$. GO-modified membranes were covered with $\mathrm{AgNO}_{3}$ solution for 10 min followed by $\mathrm{NaBH}_{4}$ solution for 5 min and all modification processes were assisted by shaking. (The detailed modification process is described in Supporting Information).

\subsection{Membrane characterization}

The distribution of AgNPs and the morphology of the pristine and functionalized membranes were observed by field-emission scanning electron microscopy (FE-SEM JEOL, JSM-7600 TFE). The roughness parameters of the membranes were determined using atomic force microscopy (AFM, Dimension 3100) in tapping mode. The elemental composition of the virgin and functionalized membranes were determined by X-ray photoelectron spectroscopy (XPS, SK-Alpha). Raman spectroscopy of pristine and modified membranes was conducted (Invia Reflex-Renishaw) to confirm the presence of GO nanosheets on the membrane surface. Surface hydrophilicity and surface energy were evaluated by contact angle measurements of DI-water, diiodomethane, and glycerol using the sessile drop method. Membrane performance tests were conducted by using a cross-flow cell in RO and FO mode (detailed procedure can be found elsewhere). ${ }^{36-37}$

\subsection{Anti-microbial properties of modified membranes}

The biocidal properties of the pristine and modified membranes were examined using the colony forming unit (CFU) counting method. The procedure is described in Supporting Information.

\subsection{Loading, stability and release of AgNPs}

The reservoir method ${ }^{16}$ was used to measure the silver ion loading, stability under physical stress ( $7 \mathrm{~min}$ bath sonication), and ions released from functionalized membranes. The detailed procedure is presented in the Supporting Information. 


\subsection{Regeneration of AgNPs on the membrane surfaces}

To study the regeneration of AgNPs, silver decorated GO-functionalized membranes were first immersed in DI water for seven days. Fresh DI water was replaced every 24 hours and the ionrelease process was accelerated by shaking $(50 \mathrm{rpm})$. AgNPs were then regenerated on the surface of the membranes using the procedure described in section 2.2 and the Supporting Information. The success of the regeneration process was confirmed by examination of antimicrobial performance, contact angle measurements, loading measurements, and XPS analysis.

\section{RESULTS AND DISCUSSION}

3.1 AgNPs were formed successfully and their loading increased in the presence of GO nanosheets

Backscattered electron (BSE) SEM images in Figure 1 indicate the presence of bright spots with strong Ag signals on the surface of the modified membranes, confirmed by EDX analysis (Figure 1). Furthermore, the AgNP size and distribution appear different for samples with and without GO nanosheets (Figure S3). In the presence of GO nanosheets, well-distributed and finer AgNPs were formed in comparison with AgNPs formed directly on the surface of pristine membranes. The AFM analysis results, as depicted in the Supporting Information (Table S1), exhibit a slight decrease in membrane roughness due to in situ AgNPs formation, while the incorporation of GO nanosheets flattened the surface and decreased surface roughness drastically.

The presence and content of metallic silver were also confirmed by studying the elemental composition of the membrane surfaces via XPS analysis. Strong signals of $\mathrm{Ag}$ (3d) in both the $\mathrm{Ag}$ and GO-Ag-functionalized membranes were clearly observed in XPS spectra (Figure 2A) thus confirming the successful formation of AgNPs. The intensity of Ag (3d) signals (centered at 373.9 and $367.9 \mathrm{eV}$ ) for GO-Ag-functionalized membranes were stronger than the signals for Ag-functionalized membranes, indicating that silver content increases in the presence of GO nanosheets.

Silver loading and release behavior were investigated more precisely by ICP-MS analysis. The results (Figure 2B and C) shows that silver loading on GO-functionalized membranes is nearly four times greater than the loading on pristine ones. Although the silver release from GO-Ag- 
functionalized membrane was greater, the lifetime of AgNPs seems to be longer in comparison with Ag-functionalized membranes. The stability of AgNPs under physical stress was also investigated and the results (Figure S3) clearly demonstrate that GO significantly increased Ag stability after seven minutes of sonication. Finally, the Ag concentration in both the feed and permeate solution was measured after 24-hour performance tests of Ag-modified and GO-Agmodified membranes in RO mode. Results for both modified membranes showed the presence of approximately $2 \mu \mathrm{g} / \mathrm{L}$ of silver on the feed side and $0.2 \mu \mathrm{g} / \mathrm{L}$ silver on the permeate side, which is negligible in comparison to the maximum contamination limit of $\mathrm{Ag}$ in drinking water (i.e., $100 \mu \mathrm{g} / \mathrm{L}$ ) as established by the World Health Organization. ${ }^{17}$

Membrane hydrophilicity and surface energy have substantial effects on membrane fouling and biofouling. ${ }^{38}$ Surfaces with higher surface energy and hydrophilicity allow the formation of a tightly bonded water molecule layer which provides a repulsive barrier against the adsorption of foulants. The Van-Oss theory as used elsewhere ${ }^{39,40}$ was employed to measure surface energy. Figure 2D represents the water contact angle and interfacial free energy of cohesion (hydrophilicity) of pristine and modified membranes. The results indicate that the incorporation of AgNPs onto both pristine and GO-modified membranes decreased their contact angle and increased their hydrophilicity. For GO-modified membranes, increased hydrophilicity can be attributed to the presence of oxygen-containing functional groups. For Ag-modified membranes, decreased contact angle may be a result of the changes of surface roughness and the presence of AgNPs which can be converted to Ag ions and alter the surface charge distribution as well as possibly due to the formation of silver oxide layer on the surface of AgNPs. For GO-Ag-modified membranes, both surface functional groups (attributed to GO) and AgNPs (presence of cations) play a role in increasing hydrophilicity. Increasing hydrophilicity can also lead to an increase in water flux around 3\% for FO and around 13\% for RO modes of operation (results are presented in the Supporting Information in Figure S2), respectively. Membranes modified with both GO and GO-Ag have a higher flux than pristine membranes while Ag-modified membranes have the same flux as pristine membranes. Moreover, membrane modification results in insignificant changes in salt rejection (around $2.5 \%$ decrement) and reverse salt flux (around $3.5 \%$ increment).

\subsection{Bacterial growth inhibition increased in the presence of $G O$ nanosheets}


The antimicrobial behavior of different membrane surfaces - pristine, GO-modified, in situ Ag-modified, and GO-Ag-modified membranes - was examined using a model, nonpathogenic E. coli strain to verify the anti-biofouling potential of the membranes. In comparison with the polyamide pristine membrane, membranes modified with GO nanosheets and in-situ-formed AgNPs exhibited 50\% and 80\% inactivation in comparison to pristine membrane, respectively (Figure 3A). The inactivation performance of GO-Ag-modified membranes was even higher. This synergetic effect is due to the combined antibacterial properties and inactivation mechanisms of the GO nanosheets and AgNPs. GO nanosheets are contact-based biocidal materials and are believed to potentially rupture the cell membranes with their sharp edges ${ }^{41-43}$ or mediate lipid peroxidation induced by the oxidative nature of GO. Releasing reactive oxygen species (ROS) or direct oxidation of cellular component also plays a role in bacterial inactivation. ${ }^{28-30,44}$ The mechanism of inactivation of $\mathrm{AgNPs}^{12}$ is being researched further, yet still remains in question. There are reports on the inactivation properties of AgNPs claiming that the nanoparticles enter the cell and selectively attack the respiratory chain, causing cell division which eventually leads to cell death ${ }^{12}$ but other contradicting reports indicating that the silver ions $\left(\mathrm{Ag}^{+}\right)$to be the major contributing factor in inactivation of microbes through reacting with the cysteine of the cell membrane ${ }^{13,45}$ These reports suggest that AgNPs may serve as a more effective $\mathrm{Ag}^{+}$delivery vehicle, whereby AgNPs of smaller size provide a larger specific surface area and result in a faster $\mathrm{Ag}^{+}$release rate as compared to larger AgNPs. Loading and release results (Figure 2B and C) indicate that GO-Ag-modified membranes present more silver on the surface and also release more silver ions $\left(\mathrm{Ag}^{+}\right)$as a function of time. Considering each mechanism of inactivation, the bacterial inactivation capability of GO-Ag would then come from higher loading, smaller AgNP size, and more $\mathrm{Ag}^{+}$ ions released.

To further evaluate the antimicrobial behavior of the modified membranes, time-dependent static (no pressure, no flow) bacterial inactivation tests were performed using three different strains of bacteria; namely E. coli D21f2 (Gram-negative and non-pathogenic), E. coli O157:H7 (Gram-negative and pathogenic) and E. faecalis (Gram-positive and pathogenic). The results show that the modified membranes exhibited strong anti-bacterial activity against the three tested bacteria, whereas for the pristine membrane, bacteria remained viable with an increasing number of CFU observed over time (Figure 3B-D). 


\subsection{AgNPs regenerated successfully on the surface of GO-Ag-modified membranes}

The regeneration of NPs after their release has always been a challenge in the use of biocidal metal NPs such as silver or copper. The ultimate lifetime of leachable nanomaterials in reallife membrane application depends on several factors such as: size, shape, and distribution of AgNPs, the nature of bonding between NPs and membrane surface, sub layer chemistry, and the $\mathrm{pH}$ and ionic strength of water. In this study, after seven days of storage in water to allow ion release to reach plateau (as observed in Figure 2C), AgNPs were regenerated on the surface of GO-Ag-modified membranes. The silver content, XPS spectra, Raman shift and antimicrobial properties of the membranes after seven days of ion-release and after regeneration are presented in Figure 4. Silver content of the modified membrane decreased by $60 \%$ after seven days of ion-release but increased back to $70 \%$ of the initial value after regeneration (Figure 4A). The XPS analysis of modified membranes exhibited the similar trend (signals of Ag3d decreased after release but increased again after regeneration) in total agreement with ICP-MS results (Figure 4C). Surface contact angle increased and surface energy decreased indicating that the membranes lost their hydrophilicity (results are not shown), yet did not deplete to the point of pristine filters. This can be attributed to the presence of the GO nanosheets which were covalently bonded to the surface. Bacterial inactivation, much like the membrane hydrophilicity, also decreased from $98 \%$ to $80 \%$ after the depletion treatment, while the membrane regained 95\% inactivation after being regenerated with AgNPs (Figure 4B). This change in bacterial inactivation is clearly linked to the Ag content of membrane surfaces. Since the regeneration was conducted after seven days which the silver leaching process was not completed, the silver content of the membranes did not reach initial values. This difference can be attributed to the differences of the surface properties of fresh GO-modified surface before Ag decoration and the surface of the modified membrane which still has 50 percent of silver from last decoration process.

The presence of GO nanosheets was confirmed by Raman spectroscopy (Figure 4D), where both characteristic D band $\left(1350 \mathrm{~cm}^{-1}\right)$ and $\mathrm{G}$ band $\left(1590 \mathrm{~cm}^{-1}\right)$ were present in all modified membranes, even for those kept in water for seven days. The existence of GO on the membrane surface after being in the water for one week was further confirmed by the XPS peak analysis of C1S (Figure S2) where the patterns and sub-peaks for these membranes resembled those of 
the GO-modified membrane and were completely different from the patterns for the pristine membrane.

\section{ASSOCIATED CONTENT Supporting Information}

Complete materials and methods information; physical stability of AgNPs (Figure S1); Performance of pristine and modified membranes (Figure S2); SEM images of modified membranes (Figure S3); XPS peak analysis of pristine and modified membranes before and after regeneration (Figure S4); and surface roughness properties of pristine and modified membranes (Table S1). This material is available free of charge via the Internet at http://pubs.acs.org.

\section{AUTHOR INFORMATION Corresponding Author}

E-mail: saifur.rahaman@ concordia.ca (M.S. Rahaman). Tel.: +15148482424, Ext. 5058 


\section{ACKNOWLEDGEMENTS}

The authors acknowledge the Natural Sciences and Engineering Research Council (NSERC) of Canada for providing financial support for this project. Adel Soroush acknowledges the support of a graduate entrance scholarship from Concordia University.

\section{Notes}

The authors declare no competing financial interest 


\section{REFERENCES}

1. Elimelech, M.; Phillip, W. A., The future of seawater desalination: energy, technology, and the environment. Science 2011, 333, 712-717.

2. $\quad$ Shaffer, D. L.; Werber, J. R.; Jaramillo, H.; Lin, S.; Elimelech, M., Forward osmosis: Where are we now? Desalination 2015, 356, 271-284.

3. MARK R. WIESNER, S. C., The promise of membrane technology. Environ Sci Technol 1999, 33, 360A-366A.

4. D. Rana, T. M., Surface modifications for antifouling membranes. Chem. Rev. 2010, $110,2448-2471$.

5. $\quad$ Matin, A.; Khan, Z.; Zaidi, S. M. J.; Boyce, M. C., Biofouling in reverse osmosis membranes for seawater desalination: Phenomena and prevention. Desalination 2011, 281, 1-16.

6. Kang, G. D.; Cao, Y. M., Development of antifouling reverse osmosis membranes for water treatment: A review. Water Res 2012, 46, 584-600.

7. Wiley, B.; Sun, Y.; Mayers, B.; Xia, Y., Shape-controlled synthesis of metal nanostructures: the case of silver. Chemistry 2005, 11, 454-463.

8. Sun, Y.; Xia, Y., Shape-controlled synthesis of gold and silver nanoparticles. Science 2002, 298, 2176-2179.

9. Thanh, N. T.; Maclean, N.; Mahiddine, S., Mechanisms of nucleation and growth of nanoparticles in solution. Chem Rev 2014, 114, 7610-7630.

10. Liao, H. G.; Niu, K.; Zheng, H., Observation of growth of metal nanoparticles. Chem Commun (Camb) 2013, 49, 11720-11727.

11. Marambio-Jones, C.; Hoek, E. M. V., A review of the antibacterial effects of silver nanomaterials and potential implications for human health and the environment. Journal of Nanoparticle Research 2010, 12, 1531-1551.

12. Li, W. R.; Xie, X. B.; Shi, Q. S.; Zeng, H. Y.; Ou-Yang, Y. S.; Chen, Y. B., Antibacterial activity and mechanism of silver nanoparticles on Escherichia coli. Appl Microbiol Biotechnol 2010, 85, 1115-1122.

13. Xiu, Z. M.; Zhang, Q. B.; Puppala, H. L.; Colvin, V. L.; Alvarez, P. J., Negligible particle-specific antibacterial activity of silver nanoparticles. Nano Lett 2012, 12, 4271-4275.

14. Ben-Sasson, M.; Lu, X.; Bar-Zeev, E.; Zodrow, K. R.; Nejati, S.; Qi, G.; Giannelis, E. P.; Elimelech, M., In situ formation of silver nanoparticles on thin-film composite reverse osmosis membranes for biofouling mitigation. Water Res 2014, 62, 260-270.

15. Madhavan, P.; Hong, P. Y.; Sougrat, R.; Nunes, S. P., Silver-enhanced block copolymer membranes with biocidal activity. ACS Appl Mater Interfaces 2014, 6, 18497-18501.

16. Mauter, M. S.; Wang, Y.; Okemgbo, K. C.; Osuji, C. O.; Giannelis, E. P.; Elimelech, M., Antifouling ultrafiltration membranes via post-fabrication grafting of biocidal nanomaterials. ACS Appl Mater Interfaces 2011, 3, 2861-2868.

17. Tang, L.; Livi, K. J. T.; Chen, K. L., Polysulfone membranes modified with bioinspired polydopamine and pilver panoparticles formed in situt to mitigate biofouling. Environmental Science \& Technology Letters 2015, 2, 59-65.

18. Tang, L.; Huynh, K. A.; Fleming, M. L.; Larronde-Larretche, M.; Chen, K. L., Imparting antimicrobial and anti-adhesive properties to polysulfone membranes through modification with silver nanoparticles and polyelectrolyte multilayers. J Colloid Interface Sci 2015, 451, 125-133. 
19. Yin, J.; Yang, Y.; Hu, Z.; Deng, B., Attachment of silver nanoparticles (AgNPs) onto thin-film composite (TFC) membranes through covalent bonding to reduce membrane biofouling. Journal of Membrane Science 2013, 441, 73-82.

20. Zodrow, K.; Brunet, L.; Mahendra, S.; Li, D.; Zhang, A.; Li, Q.; Alvarez, P. J., Polysulfone ultrafiltration membranes impregnated with silver nanoparticles show improved biofouling resistance and virus removal. Water Res 2009, 43, 715-723.

21. Liu, X.; Qi, S.; Li, Y.; Yang, L.; Cao, B.; Tang, C. Y., Synthesis and characterization of novel antibacterial silver nanocomposite nanofiltration and forward osmosis membranes based on layer-by-layer assembly. Water Res 2013, 47, 3081-3092.

22. Sawada, I.; Fachrul, R.; Ito, T.; Ohmukai, Y.; Maruyama, T.; Matsuyama, H., Development of a hydrophilic polymer membrane containing silver nanoparticles with both organic antifouling and antibacterial properties. Journal of Membrane Science 2012, 387-388, 16.

23. Daer, S.; Kharraz, J.; Giwa, A.; Hasan, S. W., Recent applications of nanomaterials in water desalination: A critical review and future opportunities. Desalination 2015, 367, 37-48. 24. Huang, X.; Qi, X.; Boey, F.; Zhang, H., Graphene-based composites. Chem Soc Rev 2012, 41, 666-686.

25. Chao Xu, X. W., and Junwu Zhu, Graphene-metal particle nanocomposites. J. Phys. Chem. C 2008, 112, 19841-19845

26. Compton, O. C.; Nguyen, S. T., Graphene oxide, highly reduced graphene oxide, and graphene: versatile building blocks for carbon-based materials. Small 2010, 6, 711-723.

27. Dreyer, D. R.; Park, S.; Bielawski, C. W.; Ruoff, R. S., The chemistry of graphene oxide. Chem Soc Rev 2010, 39, 228-240.

28. Shaobin Liu, J. K., Tingying Helen Zeng, Chen Mario Hofmann, Ehdi Burcombe, Jun Wei, Rongrong Jiang, and Yuan, Antibacterial activity of graphite, graphite oxide, graphene oxide, and reduced graphene oxide: membrane and oxidative stress. ASC NANO 2011, 5, 69716980.

29. Chen, J.; Peng, H.; Wang, X.; Shao, F.; Yuan, Z.; Han, H., Graphene oxide exhibits broad-spectrum antimicrobial activity against bacterial phytopathogens and fungal conidia by intertwining and membrane perturbation. Nanoscale 2014, 6, 1879-1889.

30. Mejias Carpio, I. E.; Santos, C. M.; Wei, X.; Rodrigues, D. F., Toxicity of a polymergraphene oxide composite against bacterial planktonic cells, biofilms, and mammalian cells. Nanoscale 2012, 4, 4746-4756.

31. Perreault, F.; Fonseca de Faria, A.; Elimelech, M., Environmental applications of graphene-based nanomaterials. Chem Soc Rev 2015, 44, 5861-5896 .

32. de Faria, A. F.; Martinez, D. S.; Meira, S. M.; de Moraes, A. C.; Brandelli, A.; Filho, A. G.; Alves, O. L., Anti-adhesion and antibacterial activity of silver nanoparticles supported on graphene oxide sheets. Colloids Surf B Biointerfaces 2014, 113, 115-124.

33. Li, C.; Wang, X.; Chen, F.; Zhang, C.; Zhi, X.; Wang, K.; Cui, D., The antifungal activity of graphene oxide-silver nanocomposites. Biomaterials 2013, 34, 3882-3890.

34. Zhang, D.; Liu, X.; Wang, X., Green synthesis of graphene oxide sheets decorated by silver nanoprisms and their anti-bacterial properties. J Inorg Biochem 2011, 105, 1181-1186.

35. de Faria, A. F.; Perreault, F.; Shaulsky, E.; Arias Chavez, L. H.; Elimelech, M., Antimicrobial electrospun biopolymer nanofiber mats functionalized with graphene oxide-silver nanocomposites. ACS Appl Mater Interfaces 2015, 7, 12751-12759. 
36. Soroush, A.; Ma, W.; Silvino, Y.; Rahaman, M. S., Surface modification of thin film composite forward osmosis membrane by silver-decorated graphene-oxide nanosheets. Environ. Sci.: Nano 2015, 2, 395-405.

37. Cath, T. Y.; Elimelech, M.; McCutcheon, J. R.; McGinnis, R. L.; Achilli, A.; Anastasio, D.; Brady, A. R.; Childress, A. E.; Farr, I. V.; Hancock, N. T.; Lampi, J.; Nghiem, L. D.; Xie, M.; Yip, N. Y., Standard methodology for evaluating membrane performance in osmotically driven membrane processes. Desalination 2013, 312, 31-38.

38. Eric M. Vrijen Hoek, S. H., Menachem Elimelech, Influence of membrane surface properties on initial rate of colloidal fouling of reverse osmosis and nanofiltration membranes. Journal of Membrane Science 2001, 185, 115-128.

39. van Oss, C. J., Development and applications of the interfacial tension between water and organic or biological surfaces. Colloids Surf B Biointerfaces 2007, 54, 2-9.

40. Hurwitz, G.; Guillen, G. R.; Hoek, E. M. V., Probing polyamide membrane surface charge, zeta potential, wettability, and hydrophilicity with contact angle measurements. Journal of Membrane Science 2010, 349, 349-357.

41. Li, Y.; Yuan, H.; von dem Bussche, A.; Creighton, M.; Hurt, R. H.; Kane, A. B.; Gao, H., Graphene microsheets enter cells through spontaneous membrane penetration at edge asperities and corner sites. Proc Natl Acad Sci U S A 2013, 110, 12295-12300.

42. Perreault, F.; Tousley, M. E.; Elimelech, M., thin-film composite polyamide membranes functionalized with biocidal graphene oxide nanosheets. Environmental Science \& Technology Letters 2014, 1, 71-76.

43. Romero-Vargas Castrillón, S.; Perreault, F.; de Faria, A. F.; Elimelech, M., Interaction of graphene oxide with bacterial cell membranes: Insights from force spectroscopy. Environmental Science \& Technology Letters 2015, 2, 112-117.

44. Franc-ois Perreault, A. F. d. F., Siamak Nejati, Menachem Elimelech, Antimicrobial properties of graphene oxide nanosheets: why size matters. ACS Nano 2015, 9, 7226-7236.

45. Agnihotri, S.; Mukherji, S.; Mukherji, S., Size-controlled silver nanoparticles synthesized over the range 5-100 $\mathrm{nm}$ using the same protocol and their antibacterial efficacy. RSC Adv. 2014, 4, 3974-3983. 

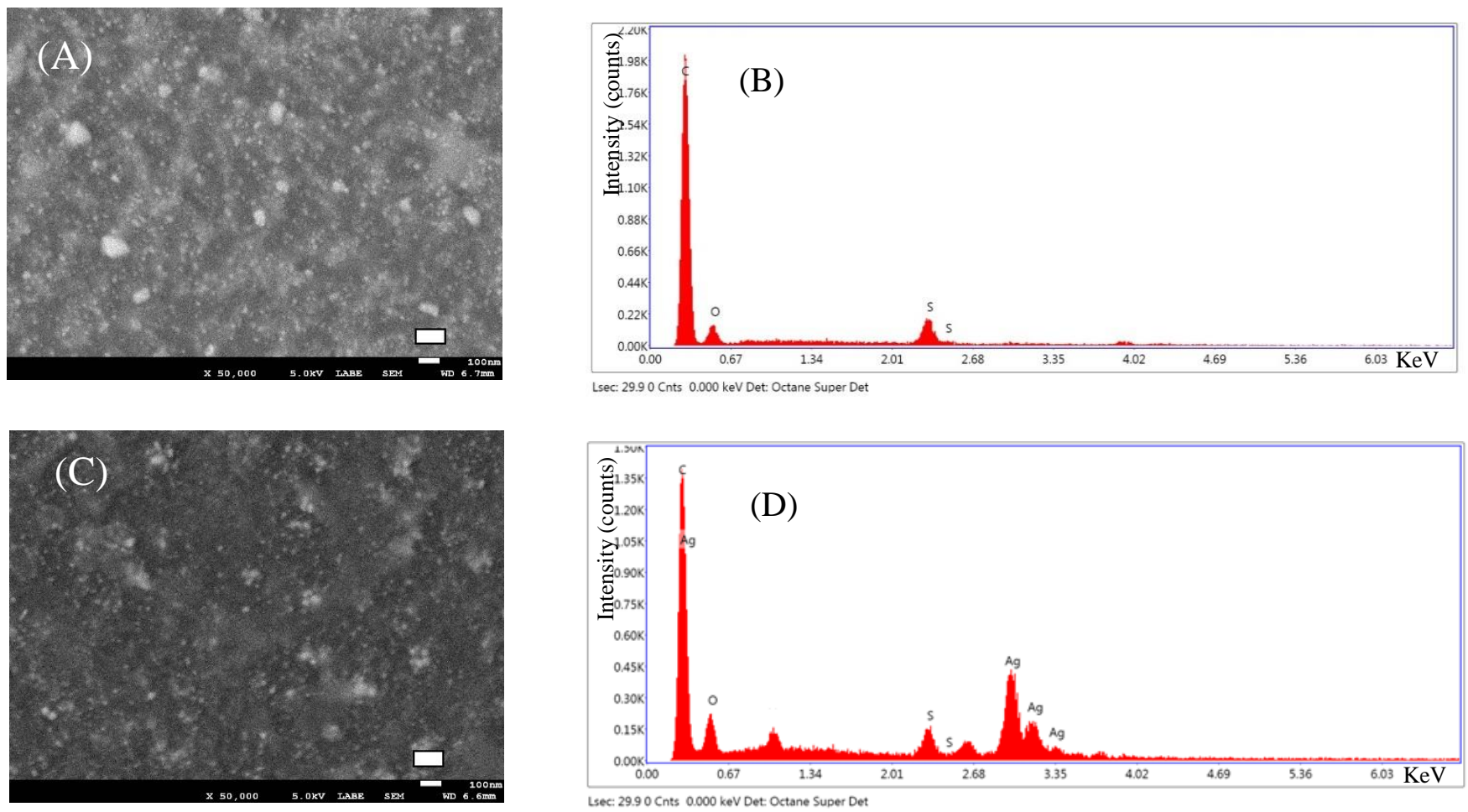

Figure 1. BSE-SEM image and EDX analysis modified membranes. BSE image of TFC-Ag in situ (A) and EDX spectrum of black spots (Polymer or GO substrate) (B), BSE-SEM image TFC-GO-Ag (C) and EDX spectrum of white spots (AgNPs) (D). The white scale bar represents $100 \mathrm{~nm}$. 

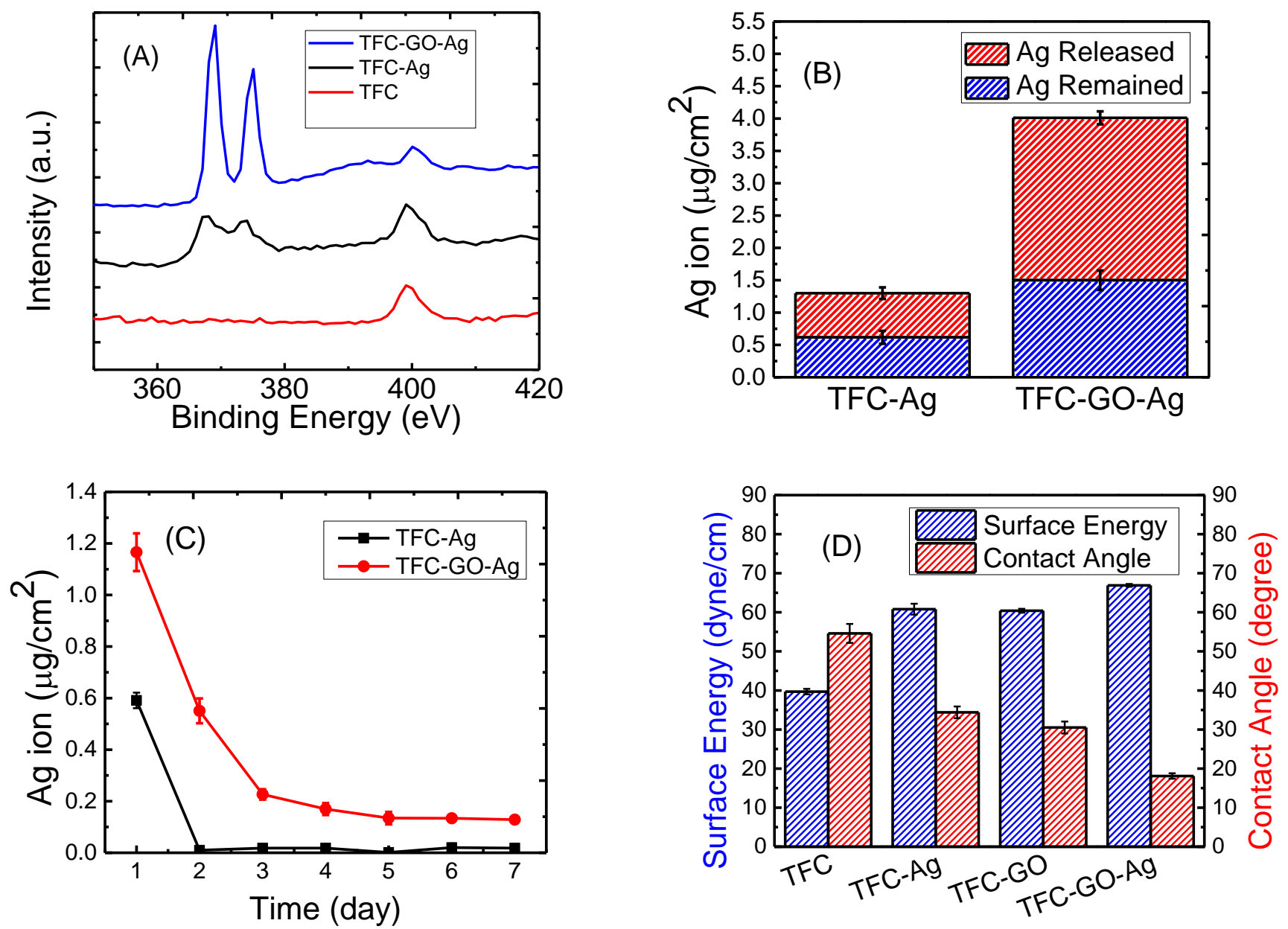

Figure 2. XPS spectra of pristine and modified membranes irradiated with a beam of monochromatic Al K $\alpha$ X-rays with $1.350 \mathrm{keV}$ of energy (A). ICP-MS results of loading and stability (B) as well as seven days of Ag release behavior in DI water (C). The modified membranes were kept in DI water for seven days immediately after modification and fresh DI water was supplied every $24 \mathrm{hr}$. Ag remained was measured by taking away of cumulative ion released during seven days from initial silver loading (ion-release procedure is presented in detail in the Supporting Information). Water contact angle and surface energy of functionalized membranes (D). 

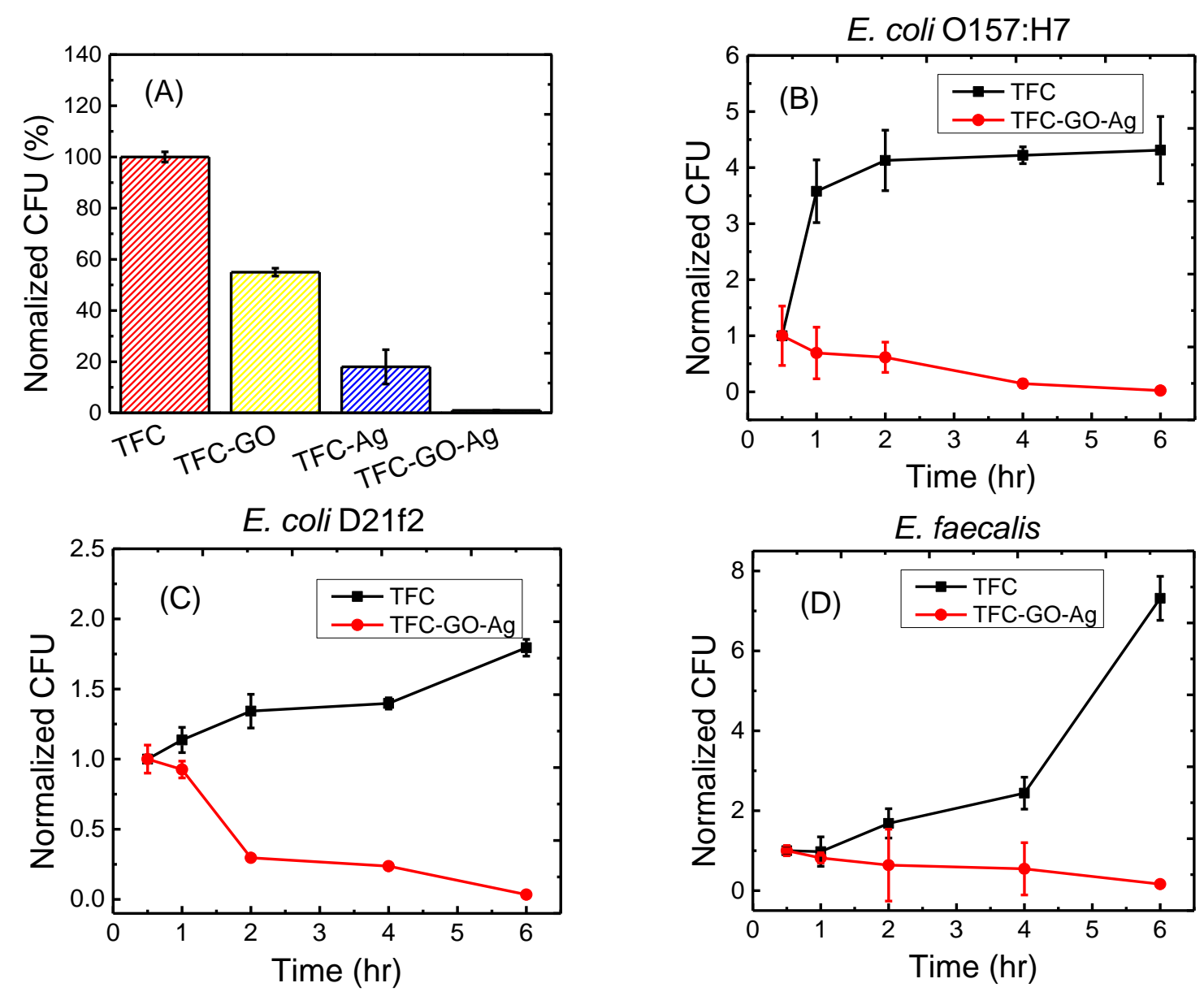

Figure 3. The percentage of culturable cells (CFU) on the Ag, GO and GO-Ag-modified surfaces after $1 \mathrm{hr}$ contact with $E$. coli D21f2 bacterial suspension in comparison with pristine membrane (A). The normalized CFU on the pristine, GO, and GO-Ag modified membranes after 0.5-6 hours contact with E. coli O157:H7 (B), E. coli D21f2 (C) and E. faecalis (ATCC 29212) (D) bacterial suspensions. For each series of membranes, CFU values were normalized by the $\mathrm{CFU}$ value at $0.5 \mathrm{hr}$ contact time. 

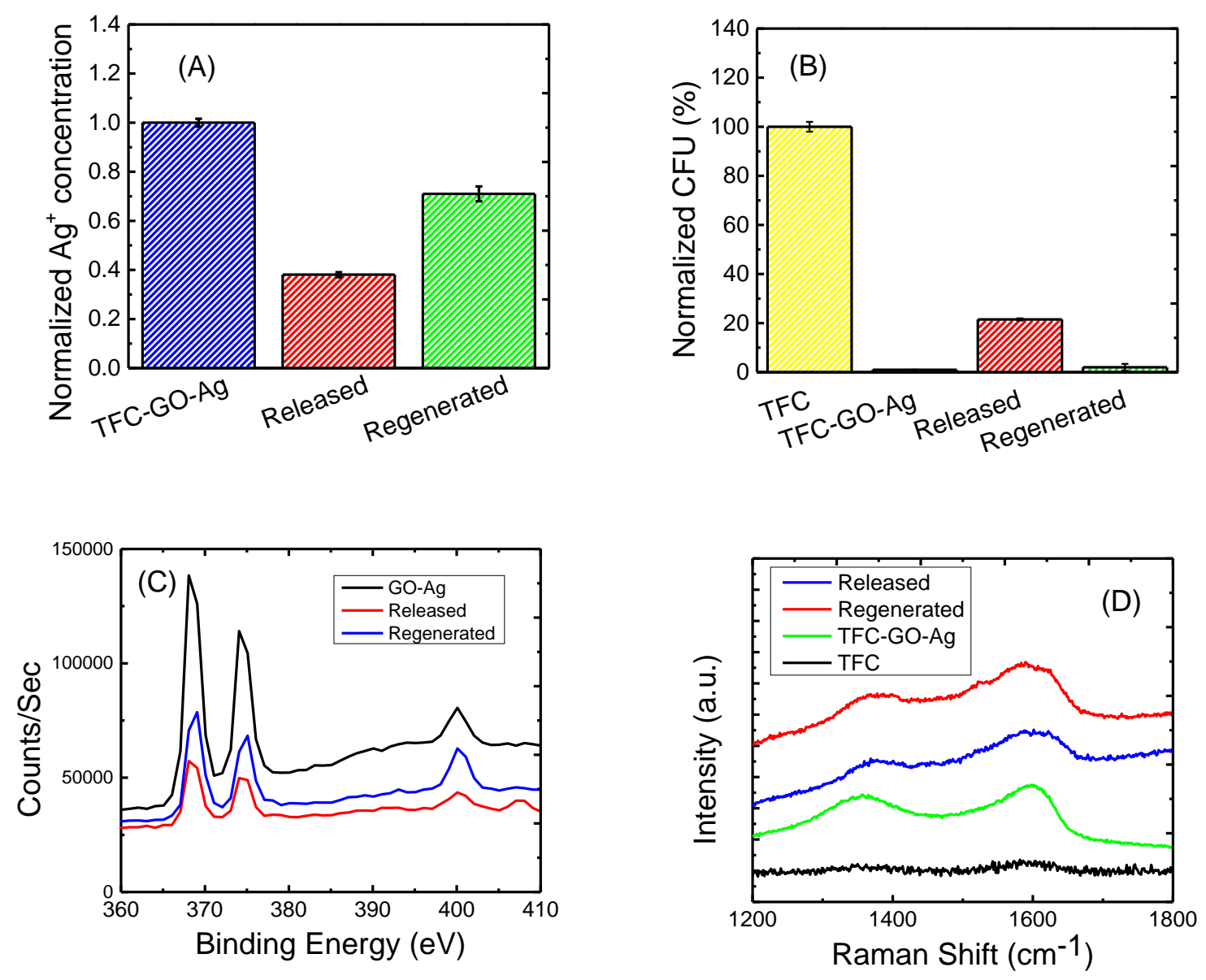

Figure 4. Normalized silver content on the surface of membranes (A), antimicrobial properties of pristine and modified membranes (B), and XPS spectra for Ag (3d) and N1S(C), Raman shift of pristine and modified membranes in $532 \mathrm{~nm}$ laser excitation (D) before and after regeneration. 


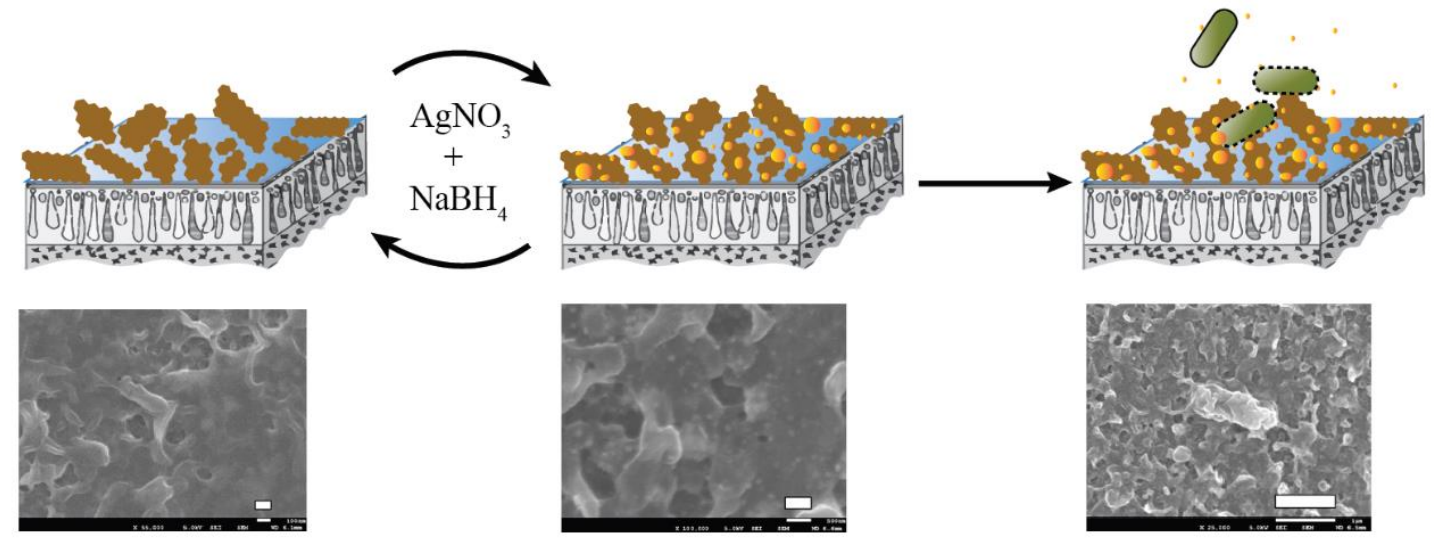

Graphical Abstract 